\title{
Propagación de onda en una interfase.
}

\section{Wave propagation at an interface}

Laura Rego Pereira. ${ }^{1}$, Amelia Beatriz Ulloa Felipe. ${ }^{2}$, Tomás Espinosa Achong. ${ }^{3}$ \& Leila Pérez Santana. ${ }^{4}$

Recibido: 15-10-2020 / Revisado: 18-11-2020 /Aceptado: 07-12-2020/ Publicado: 02-01-2021

\begin{abstract}
.
DOI: https://doi.org/10.33262/concienciadigital.v4i1.1524

Introduction. A wave is the transmission of energy without displacement of matter. It is a disturbance or agitation that moves in a certain environment and that, after passing, leaves it in its initial state. This mechanism covers a wide range of situations: From waves on the surface of a liquid to light, which is itself a type of wave. For thousands of years, waves have been the object of study. It all started when Pythagoras discovered that acoustic research was related to the theory of waves, since the strings of musical instruments generated sounds. Later, Galileo Galilei was able to relate the connection between vibratory bodies and different sounds, for which he used a pendulum, thus reaching the basic principles of MAS. Many other scientists continued their analysis, an example was Robert, who tested the theory that it is not possible for sound to travel in a vacuum, confirming that this type of wave travels in media such as air; Jean Le Rond d'Alembert also contributed his grain of sand, deriving the equation of the wave. From now on future generations are dedicated to the study of this phenomenon. Christian Huygens, in the 18th century, made a wave theory of the nature of light, deducing, some time later, that every luminous object generates a disturbance in the ether. This last experiment formed the basis for the current definition of electromagnetic wave as the form of propagation of electromagnetic radiation through space. Objective. Analyze the transmission and reflection processes of traveling waves at an interface between elastic propagation media with different densities, emphasizing the phenomenon of phase inversion by reflection and the conditions for it to occur. Methodology. For this, it was necessary to emphasize different concepts, such as wave,
\end{abstract}

\footnotetext{
${ }^{1}$ Universidad de Matanzas, Facultad de Ciencias Empresariales, Carrera de Ingeniería Industrial, Matanzas, Cuba, regolaura25@gmail.com

2 Universidad de Matanzas, Facultad de Ciencias Empresariales, Carrera de Ingeniería Industrial, Matanzas, Cuba, ulloaamelia41@gmail.com

${ }^{3}$ Universidad de Matanzas, Facultad de Educación, tomas.espinosa@umcc.cu

${ }^{4}$ Universidad de Matanzas, Facultad de Ciencias Empresariales, Carrera de Ingeniería Industrial, Matanzas, Cuba, perezleila622@gamil.com
} 
mechanical wave, traveling wave, wave motion, reflection of a traveling wave, among others. Results. All waves, regardless of their class or propagation medium, have common properties that are clearly manifested in space, passing from one medium to another with different characteristics, the same ones that will be of vital importance for the changes in speed and direction that it undergoes. the wave disturbance at the interface, so that according to the characteristics of the medium a change in some of its properties will result in the wave motion changing irremediable. Conclusions. The waves were classified according to various criteria, as well as the main elements that describe it. It was shown that when a wave changes from one medium to another, it is reflected and depending on the boundary conditions that limit it, this reflection will be inverted or not. The law of reflection for plane waves and diffraction in circular waves was also studied. Consequently, the superposition principle was explained, which addresses the resulting wave function obtained through the sum of two completely individual waves that overlap.

Keywords: traveling, reflection, boundary, condition, superposition.

\section{Resumen.}

Introducción. Una onda es la trasmisión de energía sin desplazamiento de materia. Se trata de una perturbación o agitación que se desplaza en un ambiente determinado y que, después de pasar, lo deja en su estado inicial. Este mecanismo cubre una amplia gama de situaciones: Desde las ondas en la superficie de un líquido hasta la luz, que es en sí un tipo de onda. Desde hace miles de años las ondas han sido objeto de estudio. Todo comenzó cuando Pitágoras descubrió que la investigación acústica tenía relación con la teoría de las ondas, ya que las cuerdas de los instrumentos musicales generaban sonidos. Tiempo después Galileo Galilei pudo relacionar la conexión existente entre cuerpos vibratorios y diferentes sonidos, para lo cual utilizó un péndulo, llegando así a los principios básicos del MAS. Muchos otros científicos continuaron su análisis, ejemplo fue Robert, quien probó la teoría de que no es posible que el sonido viaje en el vacío, confirmándose que este tipo de onda viaja en medios como el aire; también Jean Le Rond d' Alembert aportó su granito de arena, derivando la ecuación de la onda. De aquí en adelante las futuras generaciones se dedican al estudio de este fenómeno. Christian Huygens, en el siglo XVIII realizó una teoría ondulatoria de la naturaleza de la luz, deduciendo, tiempo más tarde, que todo objeto luminoso genera perturbación en el éter. Este último experimento constituyó la base para la definición actual de onda electromagnética como la forma de propagación de la radiación electromagnética a través del espacio. Objetivo. Analizar los procesos de transmisión y reflexión de las ondas viajeras en una interfase entre medios de propagación elásticos con diferente densidad haciendo énfasis en el fenómeno inversión de la fase por reflexión y las condiciones para que ocurra. Metodología. Para ello fue necesario enfatizar sobre 
diferentes conceptos, como el de onda, onda mecánica, onda viajera, movimiento ondulatorio, reflexión de una onda viajera, entre otros. Resultados. Todas las ondas, independientemente de su clase o medio de propagación, tienen propiedades comunes que se manifiestan claramente en el espacio, pasando de un medio a otro con características distintas, las mismas que serán de vital importancia para los cambios de velocidad y dirección que sufre la perturbación ondulatoria en la interfase, de modo que de acuerdo a las características del medio un cambio en algunas de sus propiedades traerá como consecuencia que dicho movimiento ondulatorio cambie irremediablemente. Conclusiones. Se clasificaron las ondas respecto a diversos criterios, así como los elementos principales que la describen. Se demostró que cuando una onda cambia de un medio a otro, esta es reflejada y dependiendo a las condiciones de frontera que la limitan, este reflejo será invertido o no. También fue estudiada la ley de reflexión para ondas planas y la difracción en ondas circulares. Consecuentemente se explicó el principio de superposición, el cual aborda sobre la función de onda resultante obtenida a través de la suma de dos ondas completamente individuales que se solapan.

Palabras claves: onda viajera, reflexión de una onda, condición de frontera, principio de superposición.

\section{Introducción.}

Las ondas son parte de nuestra existencia y se manifiestan en muchas escalas y con una enorme variedad de efectos. Quizás las ondas más familiares podrían ser las generadas en un estanque después de que se lanza una piedra. Otras ondas que no podemos ver son las ondas sonoras que se propagan en el aire. Permiten ambos audición y habla. Las ondas elásticas son del mismo tipo de ondas acústicas, pero también pueden ser de naturaleza transversal, ya que pueden propagarse en sólidos. A continuación, se investigará sobre los tipos de propagación que tienen las ondas, resaltando la reflexión de las mismas en una interfase. Para ello se persigue como objetivo: Analizar los procesos de transmisión y reflexión de las ondas viajeras en una interfase entre medios de propagación elásticos con diferente densidad haciendo énfasis en el fenómeno inversión de la fase por reflexión y las condiciones para que ocurra.

En física, se utiliza la palabra "onda" para designar la trasmisión de energía sin desplazamiento de materia. Se trata de una perturbación o agitación que se desplaza en un ambiente determinado y que, después de pasar, lo deja en su estado inicial. Este mecanismo cubre una amplia gama de situaciones: Desde las ondas en la superficie de un líquido hasta la luz, que es en sí un tipo de onda. (Waves Orange, 2020).

Desde hace miles de años las ondas han sido objeto de estudio de destacados científicos: 
Isaac Newton, a partir de sus experimentos, enuncio su famosa teoría de la emisión, según la cual la luz consiste en partículas materiales y los fenómenos ópticos se deben a interacciones mecánicas tales como atracciones, repulsiones, choques elásticos, etc. Newton concluyó que estas partículas son emitidas por os cuerpos luminosos y sus tamaños son diferentes para los distintos colores; y no pueden tener naturaleza ondulatoria porque se transmiten en línea recta únicamente, contraria, ente a como lo hace el sonido que si consiste en la propagación de ondas longitudinales. Además, Boyle y Hooke habían desarrollado bombas de vacío y habían demostrado que la luz, al contrario del sonido puede transmitirse por el vacío. Como las ondas necesitan un medio por el que transmitirse, Newton concluía que la luz no podía tener naturaleza ondulatoria. (López, José; et-al, 2005).

Pitágoras descubrió que la investigación acústica tenía relación con la teoría de las ondas, ya que las cuerdas de los instrumentos musicales generaban sonidos.

Galileo Galilei estudió el movimiento del péndulo y sus oscilaciones. Tuvo la idea observando el movimiento de las campanas de la catedral de Pisa, que eran mecidas por el viento. Así pues, en 1583 estudió el péndulo. Se dio cuenta de que el peso de la bola u objeto del péndulo daba igual, lo importante era la longitud de la cuerda que lo sujetara. (Rubio, 2019).

Con estos estudios llegó a los principios básicos del MAS.

Robert Hooke, en su teoría ondulatoria sostenía que la luz se propagaba instantáneamente a gran velocidad a través de vibraciones y que cada vibración generaba una esfera que crecía de forma regular. Con ello intenta explicar los colores y el fenómeno de la refracción. Además, abanderaba el planteamiento de que la luz se comporta en ondas similares a las del sonido, por lo que necesitaba un medio material para propagarse. (Flores, 2015).

D’Alembert, su primer estudio fue acerca del problema de las cuerdas vibrantes reposa en las Memoria de la Historia de la Academia de Ciencias y Bellas Letras de Berlín en los años 1747 y 1750. A principios del siglo XVII las investigaciones conciernen esencialmente al cálculo del tiempo de vibración de una cuerda tendida fija en los extremos. En la Memoria de la Historia de la Academia de Ciencias y Bellas Letras de Berlín, titulada Investigación sobre la curva que forma una cuerda tendida puesta en vibración, del año 1747, es donde D’Alembert muestra que la cuerda no formaba necesariamente una sinusoide a cada instante; D’Alembert pretende dar una solución más general ayudado por una función en dos variables.

Para D’Alembert la palabra Resolver tiene un sentido especial en toda su obra, diferente al que le damos hoy en día; él lo empleaba particularmente para los problemas de naturaleza físico-matemática. Para lograr entender el término Resolver en D’Alembert, debemos comprender la articulación de su pensamiento al relacionar las ecuaciones complementarias con las ecuaciones diferenciales parciales, añadiendo las primeras a las segundas; 
conformando así, un conjunto de ecuaciones con alas cuales busca solucionar el problema. Por lo tanto, todo el proceso abarcado por el término Resolver en la obra de D’Alambert, se ve reflejado por la sucesión de las tres fases siguientes:

- Una primera fase de puesta en ecuación que consiste en la traducción del comportamiento dinámico del sistema en forma de EDP, a través de la aplicación de los principios de mecánica.

- Una segunda fase de integración de la EDP en la cual D’Alembert parte de la EDP por sí sola y alcanza una nueva expresión general formada de funciones arbitrarias; esto lo hace con ayuda del criterio de Euler.

- Una tercera fase donde D’Alembert considera las condiciones físicas del sistema que está estudiando; en esta etapa son añadidas las ecuaciones complementarias a la expresión general que emana de la fase de integración. (Bedoya, 2014).

Christian Huygens, dio continuidad a la teoría ondulatoria de Hooke mejorándola y añadiendo ideas a dicha teoría, a partir del principio que lleva su nombre, el cual menciona que todo punto del medio 1 que llega un frente de onda puede ser considerado como fuente de onda esféricas secundaria, donde estas ondas secundarias se combinan de tal manera que se extienden en todas direcciones y determinan el frente de onda en todo instante posterior. A finales del siglo XVII, publica su Traité de la lumière, en donde a partir de dicho principio explica la reflexión y la refracción de la luz y la describe como un movimiento de la materia que se encuentra entre nosotros y el cuerpo luminoso; piensa que es análoga al sonido necesitando de un medio material para propagarse (Flores, 2015).

En el siglo XVIII realizó una teoría ondulatoria de la naturaleza de la luz, deduciendo, tiempo más tarde, que todo objeto luminoso genera perturbación en el éter. Este último experimento constituyó la base para la definición actual de onda electromagnética como la forma de propagación de la radiación electromagnética a través del espacio.

\section{Metodología.}

En el desarrollo de esta temática fue necesario analizar una serie de aspectos como lo son la clasificación de las ondas y los elementos que la describen. Para demostrar la propagación de onda en una interfase se tomó el ejemplo de una cuerda, primeramente, con extremo fijo y luego con extremo libre. En el primero de los casos la onda al llegar a la frontera se refleja en dirección contraria y sentido inverso, mientras en el segundo caso esta onda pasa a ser reflejada en dirección contraria con igual sentido. Suponga ahora que dos ondas individuales se solapan, para hallar esa función de onda que describe a dicho desplazamiento será necesario sumar las dos funciones de ondas individuales; dicho proceso es conocido como el principio de superposición. 
Para la realización de todo este análisis fue consultada la bibliografía relacionada con el tema, como artículos y libros cuyo contenido contribuyó al enriquecimiento de este trabajo.

\section{Resultados.}

Antes de continuar, debemos definir muy bien algunos conceptos claves, como lo son, Concepto de Onda, Movimiento ondulatorio.

Onda: Una onda no es más que la perturbación que se propaga a través de un medio.

Movimiento Ondulatorio: Se basa en la existencia de un ente, no material; la onda distinta a una partícula o flujo de partículas, capaz de desplazarse de un punto a otro del espacio. (Lobo, Hebert, et-al; 2013).

Elementos de una onda:

- Ciclo: Es una oscilación o viaje completo de ida y vuelta

- Amplitud: Es la distancia vertical entre dos crestas consecutivas.

- Longitud de onda: Distancia entre dos valles seguidos, suele medirse en metros.

- Elongación: Es la distancia que hay, en forma perpendicular entre un punto de la onda y la línea de equilibrio.

- Periodo (T): Es el tiempo para un ciclo completo de oscilación de la onda.

- Frecuencia (f): Es el número de oscilaciones completas descritas en la unidad de tiempo, medida en Hertz.

- Frecuencia angular (w): Representa la frecuencia en radianes por segundo. Está relacionada por la frecuencia por:

$$
w=2 \pi f=\frac{2 \pi}{T}
$$

- Rapidez de onda: Es una descripción de cuán rápido viaja una onda. Se describe por la ecuación: (Sears, et-al; 2008).

$$
v=\frac{\lambda}{T}=\lambda f
$$

Según las características del medio:

Onda Mecánica: Es una perturbación que viaja por un material o sustancia que es el medio de la onda. Al viajar la onda por el medio, las partículas que forman el medio sufren desplazamientos de varios tipos, dependiendo de la naturaleza de la onda. (Sears, et-al; 2008). 


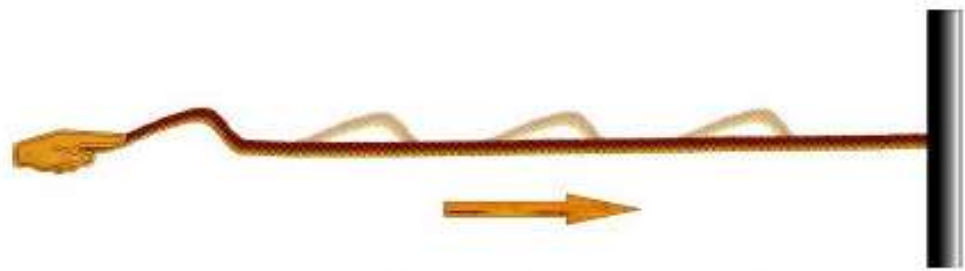

Figura 1. Onda mecánica.

Fuente: Lobo, Hebert, et-al; 2013.

Ondas Electromagnéticas: No necesitan de un medio material para propagarse, es decir, se pueden propagar el vacío, aunque el concepto de medio persiste por el requerimiento de un campo electromagnético en el espacio que al ser perturbado se propaga.

Según el número mínimo de dimensiones necesarias para representarla:

- Onda unidimensional: Son aquellas que se propagan a lo largo de una sola dirección del espacio.

- Onda bidimensional: Se propagan en dos direcciones. Pueden propagarse, en cualquiera de las direcciones de una superficie.

- Onda tridimensional: Se propagan en tres direcciones, se conocen también como ondas esféricas, porque sus frentes de onda son esferas concéntricas que salen de la fuente de perturbación expandiéndose en todas direcciones. Las ondas mecánicas y las electromagnéticas son ondas tridimensionales. (Lobo, Hebert, et-al; 2013).

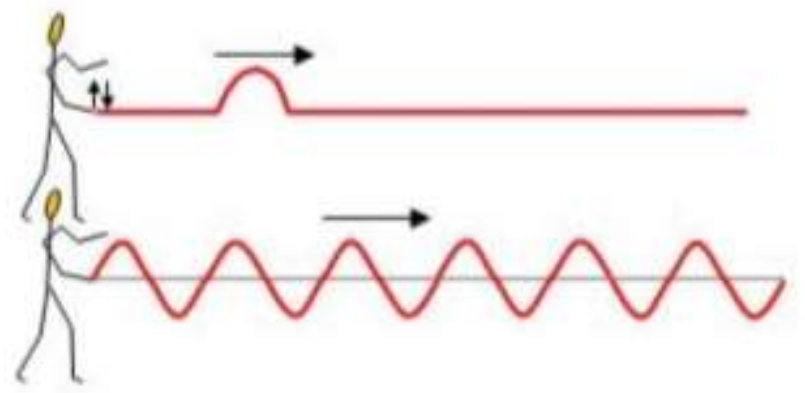

\section{Una sucesión de pulsos genera un tren de ondas, es decir, un movimiento ondulatorio.}

Figura 2. Onda unidimensional.

Fuente: Lopéz, H. A. (2006)

Según el movimiento del medio respecto a la dirección de propagación:

- Onda transversal: Son aquellas en que la vibración local del medio es perpendicular a la dirección de propagación. Si se trata de un medio material como, por ejemplo, 
una cuerda o un resorte, se podrá observar que las partículas que componen el medio oscilaran hacia arriba y hacia debajo de su posición de equilibrio, en dirección normal a la horizontal. Las ondas mecánicas son ondas transversales.

- Onda Longitudinal: La vibración de cada parte del medio es paralela a la dirección en que se propaga la onda. Es el caso de las ondas sónicas a las que se propagan a lo largo de un resorte. Si se coloca colgado un resorte, por uno de los extremos, y aplicamos un pequeño impulso, estirándolo o comprimiéndolo en dirección vertical, se producirá una oscilación vertical en el resorte, si se hace con cuidado se observará la propagación de la perturbación a lo largo del resorte. (Lobo, Hebert, et-al; 2013).

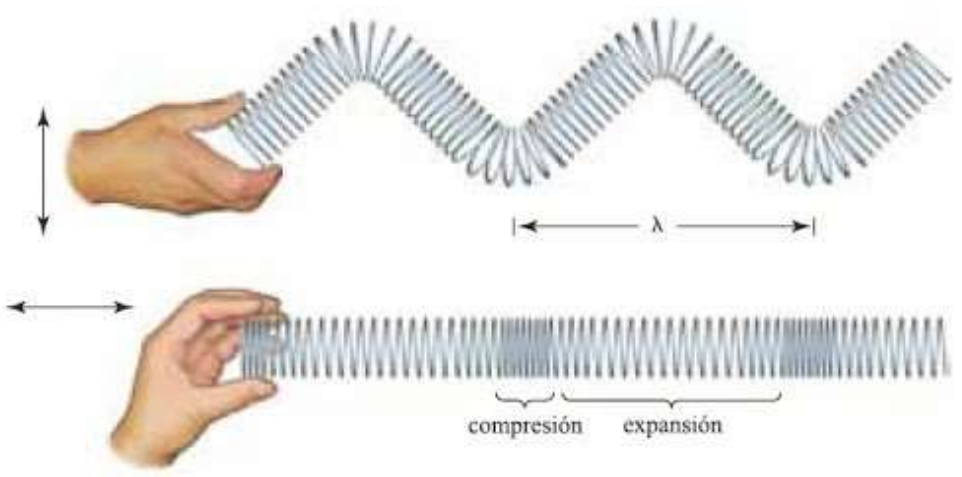

Figura 3. Onda transversal y onda longitudinal.

Fuente: Lopéz, H. A. (2006)

Nota: Con frecuencia las ondas pueden ser más bien una combinación de ondas transversales y longitudinales, como ocurre con las olas en el mar. En este caso la trayectoria seguida por una partíca de aula será una circunferencia o una elipse. (Lobo, Hebert, et-al; 2013).

Según el movimiento de su perfil: (Lobo, Hebert, et-al; 2013).

- Ondas viajeras: Cuyo perfil se mueve en el espacio, por el cual su magnitud dependerá del tiempo.

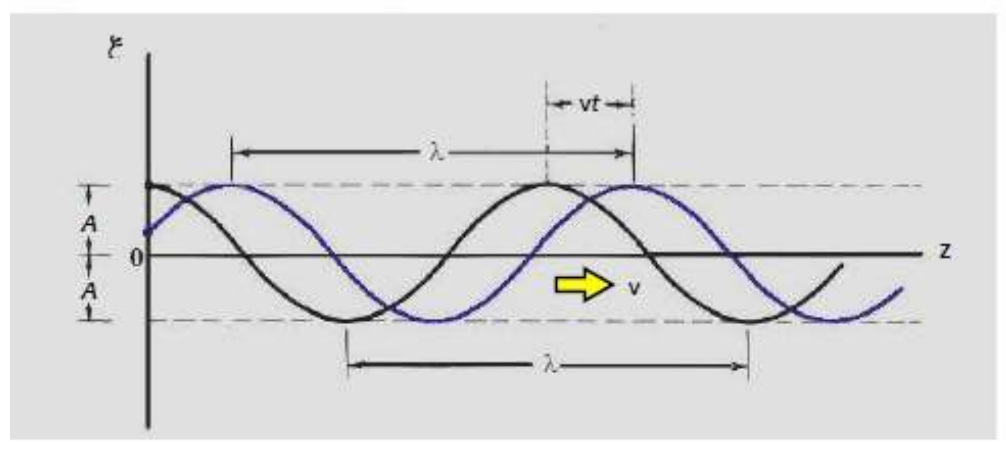

Figura 4. Onda viajera.

Fuente: Lobo, Hebert, et-al; 2013. 
- Ondas estacionarias: Su perfil no se mueve en la dirección de propagación, sino que permanece fijo o constante en cualquier instante. Surgen por la superposición de dos ondas con ciertas características particulares.
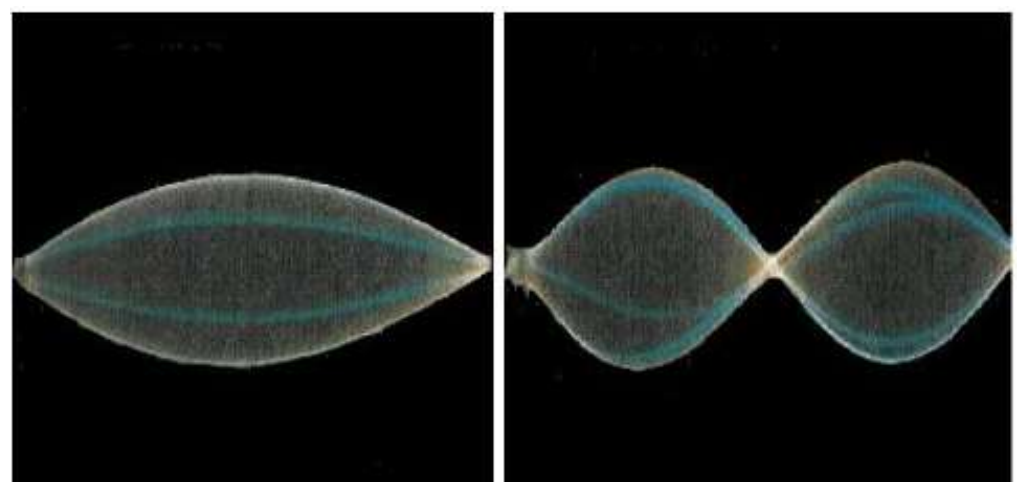

Figura 5. Onda Estacionaria

Fuente: Lobo, Hebert, et-al; 2013.

Según la forma o perfil de la onda:

- Ondas armónicas: Es aquella cuya representación gráfica de la magnitud o amplitud, en función de la coordenada espacial, conocida como perfil de la onda, es una curva senoidal o cosenoidal.

- Ondas anarmónicas: Son todas aquellas cuyos perfiles no son curvas armónicas, sin importar si permanecen constantes o no al transcurrir el tiempo. (Lobo, Hebert, et-al; 2013).

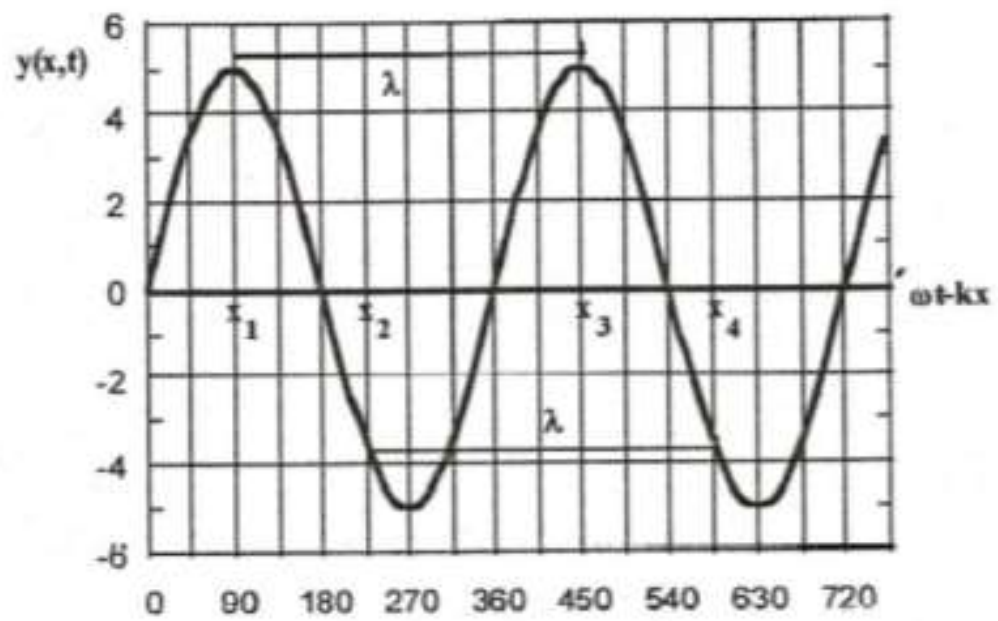

Figura 6. Onda armónica.

Fuente: Enrique Castaños. (2015) 
Según la cantidad física que represente:

- Ondas escalares: Corresponden a la representación, como un movimiento ondulatorio de cantidades físicas como la temperatura y la presión que quedan completamente especificadas por su magnitud.

- Ondas vectoriales: Cuando es necesario especificar alguna dirección aparte de la magnitud.

Propagación de Ondas:

Es muy interesante descubrir que todas las ondas, independientemente de su clase o medio de propagación, tienen propiedades comunes que se manifiestan claramente en el espacio, pasando de un medio a otro con características distintas, las mismas que serán de vital importancia para los cambios de velocidad y dirección que sufre la perturbación ondulatoria en la interfase, de modo que de acuerdo a las características del medio un cambio en algunas de sus propiedades traerá como consecuencia que dicho movimiento ondulatorio cambie irremediablemente. (Lobo, Hebert, et-al; 2013).

\section{La Ecuación de Onda}

Continuamos en esta sección restringidos a una onda que tiene una sola dirección de propagación, identificada con el eje x. Cuando encontramos la ecuación diferencial ordinaria $(\mathrm{d} 2 \mathrm{y} / \mathrm{dt} 2)+\omega 2 \mathrm{y}=0$, reconocemos inmediatamente que y corresponde al oscilador armónico simple $\mathrm{y}=\mathrm{y} 0 \operatorname{sen}(\alpha+\omega \mathrm{t}$ ). A continuación, hallaremos una ecuación diferencial para $\xi$ (puede ser otra variable), llamada la ecuación de onda, talque cuando la encontremos inmediatamente sabemos que su solución es la función de onda $\xi=\xi(\mathrm{x} \pm \mathrm{vt})$. Esta es una onda viajera que se propaga con rapidez uniforme $\mathrm{v}$, sin cambios, como una mera traslación de la función $\xi=\xi(x)$. Quiere decir que si el medio está en reposo y en algún lugar de él producimos una perturbación $\xi(\mathrm{x})$, la perturbación no se queda donde la produjimos, sino que empieza a viajar con rapidez constante y sin cambios a través del medio.

Para el oscilador armónico simple, y depende solo de una variable, t; su ecuación diferencial es ordinaria, esto es, únicamente contiene derivadas totales, y su solución es una función en particular. En cambio, cuando se propaga únicamente en la dirección $\mathrm{x}$, depende de dos variables, $\mathrm{x}$ y t; su ecuación diferencial contiene derivadas parciales respecto a x y a t, y su solución no es una función en particular, sino la familia de funciones con argumento $(\mathrm{x}-\mathrm{vt})$ ó $(\mathrm{x}+\mathrm{vt})$, compuesta por infinito número de funciones.

Con esta definición, $\xi(\mathrm{x}, \mathrm{t})=\xi(\mathrm{x} \pm \mathrm{vt})=\xi(\mathrm{u})$.Derivemos a $\xi$ respecto a $\mathrm{x}$ con la regla de derivación en cadena,

$$
\frac{\partial \xi(x, t)}{\partial x}=\frac{\partial \xi(u)}{\partial x}=\frac{\partial \xi(u)}{\partial u} * \frac{\partial u}{\partial x}
$$


Pero $\partial \xi(u) / \partial u=d \xi(u) / d u$ y $\partial u / \partial x=1$

$$
\frac{\partial \xi(\mathrm{x}, \mathrm{t})}{\partial \mathrm{x}}=\frac{\mathrm{d} \xi(\mathrm{u})}{\mathrm{du}}
$$

En consecuencia,

$$
\frac{\partial x^{2} \xi(\mathrm{x}, \mathrm{t})}{\partial x^{2}}=\frac{\mathrm{d} u^{2} \xi(\mathrm{u})}{\mathrm{d} u^{2}}
$$

Derivemos respecto a $\mathrm{t}$,

$$
\frac{\partial \xi(x, t)}{\partial t}=\frac{\partial \xi(u)}{\partial t}=\frac{\partial \xi(u)}{\partial u}=\frac{\partial u}{\partial t}
$$

Pero $\partial \xi(\mathrm{u}) / \partial \mathrm{u}=\mathrm{d} \xi(\mathrm{u}) / \mathrm{du}$ y $\partial \mathrm{u} / \partial \mathrm{t}= \pm \mathrm{v}$

$$
\frac{\partial \xi(\mathrm{x}, \mathrm{t})}{\partial \mathrm{t}}= \pm \frac{\mathrm{vd} \xi(\mathrm{u})}{\mathrm{du}}
$$

En consecuencia,

$$
\frac{\partial^{2} \xi(\mathrm{x}, \mathrm{t})}{\partial t^{2}}=\frac{v^{2} d^{2} \xi(\mathrm{u})}{\mathrm{d} u^{2}}
$$

Reemplazando la Ec. 1.16 en la anterior ecuación obtenemos la ecuación de onda unidimensional para el desplazamiento, (López; 2006).

$$
\frac{\partial^{2} y(x, t)}{\partial x^{2}}=\frac{1}{v^{2}} \frac{\partial^{2} y(x, t)}{\partial t^{2}}
$$

Reflexión y transmisión de ondas en una cuerda:

Cuando una perturbación ondulatoria se encuentra en su camino un obstáculo, o llega al límite del medio material en que se propaga, por lo menos una parte de ella es reflejada. Este fenómeno es característico de toda clase de onda, como lo son la reflexión de la luz en un espejo, con lo cual podemos ver nuestra imagen, o el eco de las ondas sonoras en un cerrado o en un acantilado. (Lobo, Hebert, et-al; 2013).

Para demostrar más claramente este fenómeno imagine una cuerda estirada. ¿Qué sucederá cuando un pulso de onda llegue al extremo de la cuerda? 


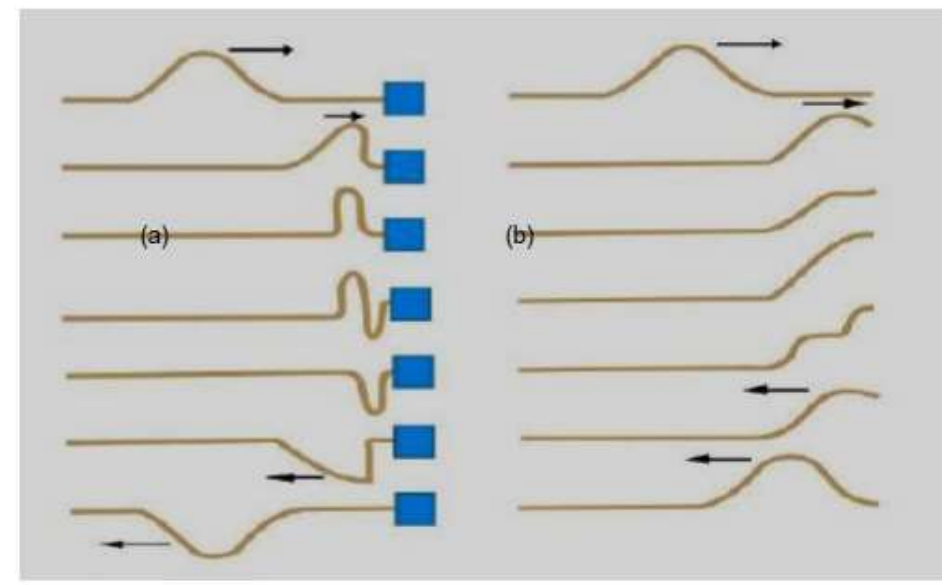

Figura 7. Reflexión de una en una cuerda a-) cuando el extremo está fijo, b-) cuando el extremo está libre

Fuente: Lobo, Hebert, et-al; 2013.

Si el extremo está sujeto a un soporte rígido, es un extremo fijo que no puede moverse, por lo que el pulso reflejado en el extremo fijo cambia no solo de dirección, sino que se refleja invertido. La explicación es muy simple a partir de las leyes de Newton, tomando en cuenta que el pulso al llegar al extremo empotrado ejerce una fuerza hacia arriba sobre el soporte, el cual a su vez ejerce otra igual, pero de sentido contrario sobre la cuerda, produciendo el pulso reflejado invertido.

Ahora, suponiendo una situación opuesta, un extremo libre que puede moverse sin resistencia en la dirección perpendicular a la longitud de la cuerda. En este caso no hay soporte, y el extremo libre se desplaza verticalmente hacia arriba un poco más que la amplitud del pulso, ejerciendo con ello un latigazo o tirón hacia arriba de la cuerda, por lo cual, reaparece el pulso en sentido contrario, pero sin invertirse. Este pulso reflejado debe tener menor amplitud, debido a la perdida de energía en la interacción con el soporte.

Estas condiciones en el extremo de una cuerda se denominan condiciones de frontera.

Este solapamiento es similar al de dos pulsos que viajan en direcciones opuestas. Al solaparse los pulsos y pasarse mutuamente, el desplazamiento total de la cuerda es la suma algebraica de los desplazamientos en este punto. Así, el movimiento de la mitad derecha de la cuerda seria el mismo si cortáramos la cuerda en el punto de intersección, y sostuviéramos el extremo fijo. Así los dos pulsos del lado derecho corresponden a los pulsos incidente y reflejado, cambiándose de modo que el desplazamiento total en $\mathrm{O}$ siempre es cero. (Sears, et-al; 2008). 
Reflexión y refracción de ondas planas:

En las ondas del tipo tridimensional o bidimensional, es de suma importancia conocer el frente de onda, que no es más que una cresta de onda en toda su extensión, como por ejemplo una ola del mar. Una línea de onda trazada la dirección de propagación se llama rayo. Es importante conocer que cuando los frentes de onda están lo suficientemente lejos de la fuente de la perturbación, comienzan a perder curvatura y se convierten en un frente de ondas planas.

En este caso, la reflexión sigue la conocida "ley de reflexión" (el ángulo que forma la onda incidente con la superficie reflectante es igual al ángulo que forma con ella la onda reflejada). La refracción de las ondas, como en la transmisión, implica un cambio de velocidad de fase por la cual, dado que la frecuencia permanece inalterada, se modificará naturalmente la longitud de onda. (Lobo, Hebert, et-al; 2013).

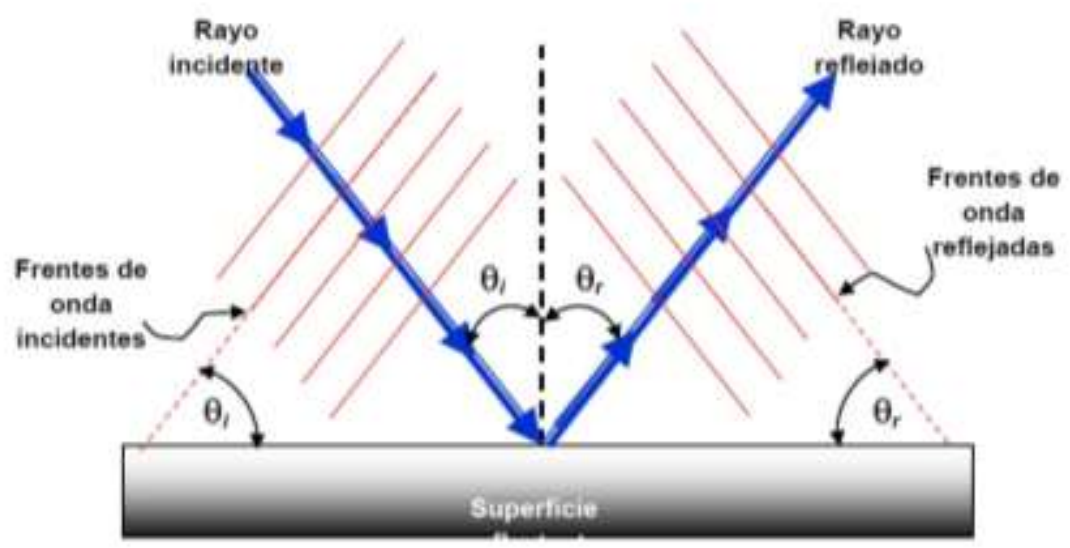

Figura 8. Ley de Reflexión

Fuente: Lobo, Hebert, et-al; 2013.

\section{Difracción de ondas circulares:}

La difracción es la desviación que sufren las ondas alrededor de los bordes y esquina que se produce cuando una porción de un frente de ondas se ve cortado o interrumpido por una barrera u obstáculo. El esquema de la onda resultante puede calcularse considerando cada punto del frente de onda original como una fuente puntual de acuerdo con el principio de Huygens y calculando el diagrama de interferencia que resulta de todas estas fuentes. (Lobo, Hebert, et-al; 2013).

\section{Principio de superposición:}

Combinar los desplazamientos de los pulsos individuales en cada punto para obtener el desplazamiento real es un ejemplo del principio de superposición: cuando dos ondas se solapan, el desplazamiento real de cualquier punto de la cuerda en cualquier instante se 
obtiene sumando el desplazamiento que tendría el punto si solo estuviera presente la primera onda y el que tendría si solo estuviera presente la segunda.

Esta ecuación es lineal, solo contiene la función y $(x, t)$ a la primera potencia. Por tanto, si dos funciones satisfacen la ecuación de onda por separado, su suma también la satisface y es un movimiento físicamente posible. Es súper importante este principio para todo tipo de ondas, si un amigo nos habla mientras escuchamos música, podemos distinguir el sonido de su voz del sonido de la música. Esto es precisamente porque la onda sonora total que llega a nuestros oídos es la suma algebraica de la onda producida por la voz del amigo y la producida por los altavoces. (Sears, et-al; 2008).

El fenómeno surgido de la superposición de dos o más ondas de cualquier clase se conoce como "interferencia", que puede ser "constructiva" y "destructiva" de acuerdo a si la amplitud de la onda resultante sea mayor o menor, respectivamente. Las frecuencias para las cuales se reproducen ondas estacionarias son la frecuencia natural y las frecuencias resonantes de la cuerda y, los distintos modos de vibración, se conocen como modos de vibración resonante. Cuando una cuerda produce ondas estacionarias está vibrando en el mismo lugar, y a las frecuencias resonantes no se necesita mucha energía para alcanzar una amplitud grande. Por ello, aunque en la cuerda pulsada aparecerán ondas viajeras en ambos sentidos, las cuales se reflejan en los extremos empotrados y regresan en sentido contrario, la mayoría de ellas interferirán y desaparecerán rápidamente, excepto aquellas con frecuencia resonante, que en la medida que sea mayor generan nuevos nodos, dando origen a los llamados modos de resonancia. La frecuencia mínima se llama frecuencia fundamental y corresponde a media longitud de onda $L=\lambda 1 / 2$, donde $\lambda=1$ es la frecuencia fundamental. Las otras frecuencias naturales se llaman sobretonos, cuando son múltiplos enteros de la fundamental se denominan armónicos, y al fundamental primer armónico, al siguiente segundo armónico o primer sobretono. (Lobo, Hebert, et-al; 2013).

\section{Aplicaciones de las ondas.}

Las aplicaciones de las ondas en la vida del ser humano se han manifestado de variadas y disímiles formas

- En la música, como bien descubrió Pitágoras, permite la producción del sonido en los instrumentos musicales y sistemas de afinación de la escala.

- En la arquitectura intervienen en el diseño de las propiedades acústicas de un local a efectos de fidelidad de la escucha, como de las formas efectivas de aislar del ruido los locales habitados.

- En la medicina se destaca en diferentes ramas como en la ecografía, ultrasonografía o ecosonografía. Y en la litotricia, técnica utilizada para destruir los cálculos que se forman en el riñón, la vejiga, los uréteres o la vesícula biliar.

- Microondas: Se denomina así debido a que se encuentra entre el rango de frecuencias comprendidas entre $1 \mathrm{Ghz}$ y $300 \mathrm{Ghz}$, su longitud es muy pequeña. Estas generan color 
cuando inciden sobre un cuerpo orgánico debido a que estos contienen moléculas de agua, actuando como dipolos magnéticos cuando una señal de microondas incide sobre estas moléculas, estas comienzan a vibrar con la misma frecuencia que las del microondas, produciendo un rozamiento entre las moléculas lo cual genera calor.

- Radiación Infrarroja: Los rayos infrarrojos se utilizan en los equipos de visión nocturna cuando la cantidad de luz visible es insuficiente para ver objetos. La radiación se recibe y después se refleja en una pantalla. Los objetos más calientes se convierten en los más luminosos.

- Frecuencias lumínicas: A cada color le corresponde una longitud de onda distinta, el azul le corresponde la menor y al rojo la mayor, los demás colores tienen una longitud entre estos valores.

- Radiación Ultravioleta: se utiliza para la desinfección del agua, del aire, de superficies. Se utilizan en la Fotoquímica, Fotoluminiscencia.

- Rayos X: Utilizados en la medicina en tomografías para crear múltiples imágenes transversales del cuerpo a modos de capas que juntas proporcionan una imagen 3D; en los aeropuertos con escáner de rayos X para el control de pasajeros que permite encontrar armas, drogas, explosivos. (Rossmann,s.f).

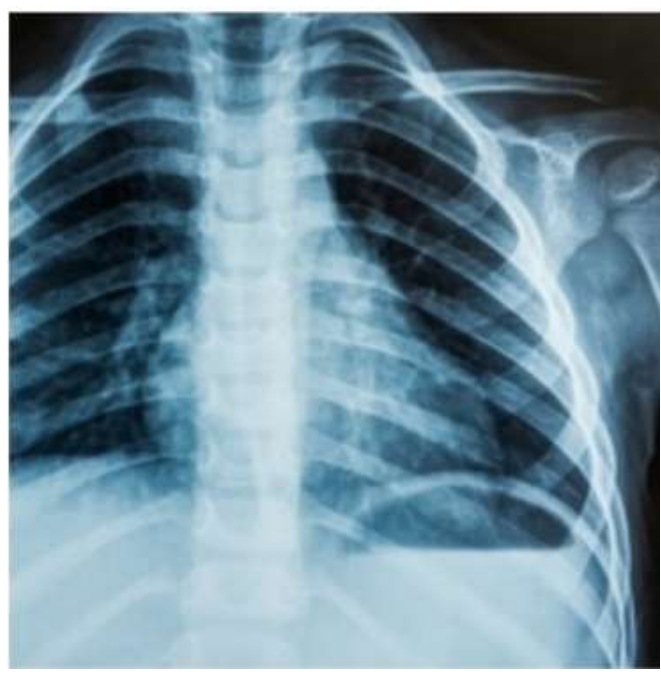

Figura 9. Rayos X.

Fuente: Enrique Castaños. (2015)

\section{Conclusiones.}

- Se definió el concepto de onda y de movimiento ondulatorio, así como se expusieron los principales elementos de las ondas (ciclo, amplitud, longitud de onda, elongación, periodo (T), frecuencia (f), frecuencia angular (w), rapidez de onda) y su clasificación según las características del medio (onda mecánica y electromagnética), dirección de propagación (onda transversal y longitudinal), movimiento de su perfil (ondas viajeras y estacionarias), entre otros aspectos. 
- Se demostró que cuando una onda cambia de un medio a otro, esta es reflejada y dependiendo a las condiciones de frontera que la limitan, este reflejo será invertido o no.

- Se analizaron los procesos de transmisión y reflexión de las ondas viajeras en una interfase, obteniéndose que cuando una perturbación ondulatoria se encuentra en su camino un obstáculo, o llega al límite del medio material en que se propaga, por lo menos una parte de ella es reflejada. También, se abordó sobre la ley de reflexión para ondas planas y la difracción en ondas circulares.

- Se explicó el principio de superposición como resultado del solapamiento simultáneo entre dos ondas de igual naturaleza.

\section{Referencias bibliográficas.}

Ariz, A. d. (s.f.). Obtenido de http://lasondasjosemaria.epiz.com

Bedoya, O. L. (2014). Ecuación de D’Alembert, de la cuerda vibrante bajo la teoría de Lie. Disponible en: https://www.core.ac.uk. Bogotá, Colombia.

Enrique Castaños. (2015). ¿Qué son las ondas? http://www.cienciaonthecrest.com

FJ, S.-S. (2017). Modeling and inversion of the microtremor H/V spectral ratio: physical basis behind the diffuse field approach. Obtenido de https://doi.org/10,1186/s40623-017-0667-6.

Flores, O. (2015). La naturaleza de la luz, corpúsculos y ondas. Obtenido de http://www.luz2015.unam.mx

José María López Sancho, Esteban Moreno Gómez, María José Gómez Díaz. (2005). La naturaleza de la luz. Obtenido de http://www.museovirtual.csic.es

La Guía de Física. (2013). Ondas armónicas. http://www.fisica.laguia2000.com Lobo, Hebert; et-al. (2013). Mecanica del Movimiento Ondulatorio. Material de apoyo teorico, Departamento de Fisica y Matematica, Universidad de los Andes, , Trujillo, Venezuela. Obtenido de http: Ilwww.grincef.nurr.ula.ve

Lopéz, H. A. (2006). Física de las ondas. Universidad de Antoquia, Instituto de Física, Medellín. Obtenido de http://www.aprendeenlinea.udea.edu.com

Marcelo Alonso, Edward J. Finn. (1976). Física. Fondo Educativo Interamericano. ISBN 84-0320234-2.

MedlinePlus. (2020). Rayos X. Biblioteca Nacional de Medicina de los EE.UU. http://www.medlineplus.gov 
OKDIARIO. (2016 19 de abril). ¿Sabes qué es el efecto Doppler y para qué sirve? [ Web $\log$ post]. Disponible en:

http://www.google.com/amp/s/okdiario.com/curiosidades/que-efecto-doppler58278/amp

Rossmann, R. A. (s.f.). ASDOPEN. Obtenido de ASDOPEN:

http://www.asdopen.unmsm.edu.pe

Rubio, N. M. (2019). Psicologia y Mente. Obtenido de http://www.12aportacionesdegalileogalilei.com

Sears, et-al. (2008). Física Universitaria (Vol. I). La Habana: Félix Varela.

Paulini, Ramón. (2020 24 de marzo). Las ondas. [ Web log post].Recuperado el 31 de octubre de 2020, de https://radio-waves.orange.com

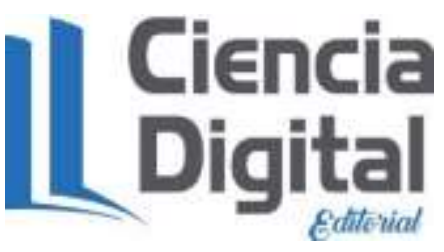


PARA CITAR EL ARTÍCULO INDEXADO.

Rego Pereira, L., Ulloa Felipe, A. B., Espinosa Achong, T., \& Pérez Santana, L. (2021). Propagación de onda en una interfase. ConcienciaDigital, 4(1), 48-65. https://doi.org/10.33262/concienciadigital.v4i1.1524

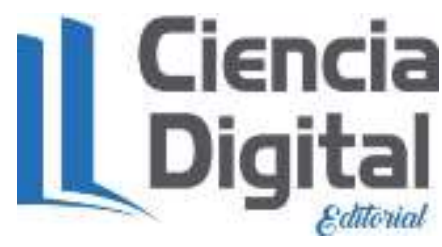

El artículo que se publica es de exclusiva responsabilidad de los autores y no necesariamente reflejan el pensamiento de la Revista Conciencia Digital.

El artículo queda en propiedad de la revista y, por tanto, su publicación parcial y/o total en otro medio tiene que ser autorizado por el director de la Revista Conciencia Digital.

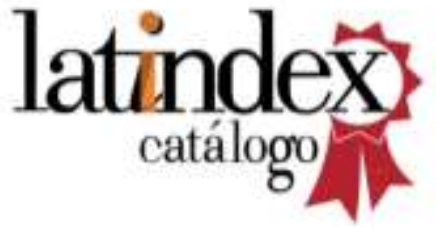

